This item was submitted to Loughborough's Research Repository by the author.

Items in Figshare are protected by copyright, with all rights reserved, unless otherwise indicated.

\title{
Conductive PANI fibres and determining factors for the electrospinning window
}

\section{PLEASE CITE THE PUBLISHED VERSION}

http://dx.doi.org/10.1016/j.polymer.2015.08.039

\section{PUBLISHER}

(C) Elsevier

\section{VERSION}

SMUR (Submitted Manuscript Under Review)

\section{PUBLISHER STATEMENT}

This work is made available according to the conditions of the Creative Commons Attribution-NonCommercialNoDerivatives 4.0 International (CC BY-NC-ND 4.0) licence. Full details of this licence are available at: https://creativecommons.org/licenses/by-nc-nd/4.0/

\section{LICENCE}

CC BY-NC-ND 4.0

\section{REPOSITORY RECORD}

Moutsatsou, Panagiota, Karen Coopman, Martin Smith, and Stella Georgiadou. 2015. "Conductive PANI Fibres and Determining Factors for the Electrospinning Window". Loughborough University. https://hdl.handle.net/2134/18944. 


\title{
Conductive PANI Fibres and Determining Factors for the Electrospinning Window
}

\author{
Panagiota Moutsatsou $^{a}$, Karen Coopman ${ }^{a}$, Martin B. Smith $^{b}$, Stella Georgiadou $^{a}$ \\ a Department of Chemical Engineering, Loughborough University, Leicestershire, UK \\ LE11 3TU \\ ${ }^{\mathrm{b}}$ Department of Chemistry, Loughborough University, Leicestershire, UK \\ LE11 3TU
}

\begin{abstract}
Polyaniline doped with CSA / PEO conductive nanofibres were produced by electrospinning. The electrospinning window was determined by using a three level, full factorial experimental design. The combined effects of the humidity, voltage and flow rate on the fibre morphology and diameter were examined demonstrating that the ambient humidity is the critical factor affecting the electrospinning process and determining the electrospinning window for a conductive polymer. Low humidity favors the formation of defect free fibres while high humidity either hinders fibre formation or causes the formation of defects on the fibres either due to jet discharge or due to water absorption and phase separation. High level of doping with CSA led to the formation of crystalline structures. Data fitting was used to explore the behavior of conductive polymers in electrospinning and very good agreement between experimental and theoretical predictions was obtained for only a limited range of experimental conditions, whereas deviation was observed for all other sets of conditions.
\end{abstract}

Keywords: electrospinning process parameters, conductive polymers, polyaniline, humidity

\section{Introduction}

Conductive polymers are organic polymers exhibiting electrical, optical and magnetic properties similar to those of metallic materials but with the characteristic processability and mechanical properties of polymers [1]. Their conjugated structure, meaning the alternation between the single and double bonds, favors the molecule's stability, as well as electron mobility and transport of electric charge within and between the polymer chains. Their electrical conductivities can be increased by many orders of magnitude from $10^{-10}-10^{-5}$ to $10^{2}-10^{5} \mathrm{~S} / \mathrm{cm}$ upon doping, which cover the whole insulator-semiconductor-metal range. Due to this unique nature as well as the reversible doping/dedoping process and their controllable chemical and electrochemical properties, a variety of conductive polymers, especially their 1D nanostructures such as nanotubes and nanowires, have been studied in the field of nanotechnology (nanotubes and nanowires for supercapacitors, gas sensors, energy storage, nanodiodes, actuators) and more recently they have been successfully tested as biosensors and for their ability to promote cell adhesion, regulate and modulate cell differentiation, migration, protein secretion and DNA synthesis [2,3].

Compared to other conductive polymers, polyaniline presents significant advantages such as ease of synthesis, low cost of aniline monomer and good stability in environmental conditions $[4,5]$. As all 
conductive polymers though, exactly because of its conjugated structure, it is generally insoluble in common organic solvents. Its emeraldine salt though, which is produced by doping the emeraldine base form with a suitable acid, can render the polyaniline soluble in some organic solvents [5]. In terms of electrospinnability, the small molecular weights that it is usually available at, combined with its low solubility in common organic solvents make it necessary to use blends of polyaniline with commonly electrospun insulating polymers so as to produce nanofibres [6].

PEO is a polymer that when blended with polyaniline can successfully give, bead free, homogeneous nanofibres. It also presents the advantage of availability in very high molecular weights which is very convenient in this case, since, the higher the molecular weight, the more the chain entanglements that are necessary to guarantee electrospinnability. Therefore, less mass is needed in the blend, allowing higher ratio of polyaniline in the final mat rendering the formentioned advantages of polyaniline more evident in the electrospun mat [4].

The morphology of the electrospun fibres is influenced by a variety of factors including solvent properties (dielectric constant, volatility), solution properties (viscosity, surface tension, conductivity, polymer concentration and molecular weight, incorporation of additives such as salts or surfactants), environmental conditions (temperature, humidity) and process parameters (applied voltage, flow rate, needle tip to collector distance, needle diameter, type and size of collector). Humidity has been acknowledged as one of the environmental parameters, but is not always taken into account in the electrospinning models as a significant parameter affecting the process On the contrary, it seems to be categorized as a minor parameter affecting the final jet diameter [7-11].

Studies on the effect of humidity on electrospun fibres have shown contradicting results. Electrospinning of cellulose acetate (CA) exhibited increase in nanofibre diameter with increasing humidity, while Poly-vinylpyrrolidone (PVP) showed the opposite trend. In PVP, the absorption of the surrounding water in higher relative humidity causes slower solidification of the jet, longer elongation time and as a result smaller fibre diameters. However, at high $\mathrm{RH}$ above $60 \%$, PVP nanofibres begin to fuse, probably due to loss of surface charges, resulting in apparently larger diameters. In the case of $\mathrm{CA}$, as relative humidity increased, more absorption of water caused faster precipitation and therefore, larger fibre diameters [12,13]. Pelipenko et al. also concluded that higher environmental humidity in general, results in thinner average nanofibre diameter but with higher values of relative standard deviation, after having examined a series of single polymer solutions and polymer blends. However, above a certain threshold of relative environmental humidity, which differs regarding the polymer, only beaded nanofibres are collected. Even if in all the solutions studied, the general trend of the way the relative humidity affects the process was the same, there are discrepancies regarding the thresholds and quantitative results which they attributed to different polymer characteristics [14]. High humidity has also been reported to cause fibre breakage and loss of poly-ethylene glycol fibre morphology, as a result of increased water absorption [15].

High relative humidity can also cause surface pore formation on the electrospun fibres, as it was demonstrated on PCL fibres by Nezarati et al., possibly through vapor-induced phase separation. It is also expected that porosity and pore diameter tend to increase with further increase of relative humidity. In the case of electrospinning poly-carbonate urethane, fibre collection dropped at high humidity, most likely due to increased electrostatic discharge. In the same study, humidity below $50 \%$ 
resulted in fibre breakage due to decreased electrostatic discharge from the jet for all three polymers that were electrospun (Polyethylene glycol, poly carbonate urethane and polycaprolactone) $[13,15,16]$. Tripatanasuwan et al. concluded that there is a linear decrease of the diameter of PEO fibres with increasing humidity. It was also found that above $50 \%$ of relative humidity, beaded fibres are collected, with the size of the beads systematically changing with further increase of humidity [16].

Generally, the ambient relative humidity may affect the electrospinning process in three ways. First, it may affect the solvent evaporation rate, with high humidity causing slower solidification of the jet and therefore causing the jet to elongate longer, producing thinner fibres. Very high humidity can cause the solvent to not fully evaporate throughout the process $[12,13,16]$. Secondly, as water vapor is electrically conductive, it affects the charge distribution on the Taylor cone and on the elongated jet, mainly by removing charges from the jet. In this case the surface charge density decreases leading to the formation of fibres with larger diameters. Finally, water absorption on the jet may induce polymer precipitation and phase separation changing the morphology of the fibres. These findings highlight that the effects of relative humidity on electrospun fibre morphology are dependent on polymer chemical structure and hydrophobicity, solvent miscibility with water, and solvent volatility and the results are not always predictable. Especially with regards to the electrospinning of conductive polymers, the role of humidity has not been fully explored neither understood yet. Despite their numerous potential applications, very few studies have been reported on electrospinning of conductive polymers. Sisi Li et al., studied through a wide lens, the process and solution parameters as well as environmental factors on the electrospinning of PANI-PEO blends. Two humidity conditions, $38 \%$ and $60 \%$, were directly compared and it was found that at high relative humidity ( $>60 \%)$ no fibres were formed. Low flow rate and high applied voltage appeared to have a synergistic effect to the stretching of the jet and the production of thinner nanofibres [17].

Contradicting results have also been reported with regards to the effect of the applied voltage. Zhou et al., reported that increasing the applied voltage contributes to the refinement of the fibres but enhances the phenomenon of broken filaments. Li et al., however reported that higher applied voltage resulted in bigger average diameter [4]. The exact relationship between voltage and fibre diameter is still quite vague and interdependent with other parameters such as solution properties. It has also been reported that, higher electric field can result in increased bead formation, mainly due to jet instability caused by the disruption of the shape of the Taylor cone $[10,13,18]$.

As for the flow rate, there is consensus, generally, that the increase in flow rate can result in increased formation of beads and this seems to be valid for PANI-PEO blends as well. Low flow rate, on the other hand, usually results in nanofibres of smaller diameter $[10,15,17]$.

The aim of this study is to conduct a systematic experimental study in order to determine the electrospinning window of a conductive polymer solution, to determine the combined effect of major process and environmental parameters on the behavior of a conductive polymer jet and compare the behavior of a conductive polymer during electrospinning with that of non conductive polymers reported in the literature. The effect of the level of doping on the fibre morphology will also be discussed. 


\section{Materials and Methods}

Polyaniline emeraldine base (PANI, $M w=50000),(1 R)-()-10$-camphor-sulfonic acid (HCSA), poly(ethyleneoxide) (PEO, Mw=2 000 000) and chloroform were purchased from SigmaAldrich Inc. and used without further purification.

\section{Solution Preparation}

Camphorsulfonic acid was first fully dissolved in chloroform. An adequate amount of polyaniline base was dispersed in the solution. It was first sonicated for 5minutes and kept under magnet stirring overnight. Sonication was repeated twice more during the stirring time and the solution was filtered through a $0.45 \mathrm{um}$ PTFE syringe filter. Polyethylene oxide was then added to the solution which was stirred for an additional 4 hours. Three blends of different doping level, 20\%, 60\% and 100\% were chosen to be used for electrospinning. The doped polyaniline concentration was kept the same for all solutions. Gravimetric measurements of the filters were performed to determine the final PANI content and a solution of final concentration 1,8\% w/v doped PANI/PEO (ratio 50:50) was produced for electrospinning. The surface tension of the final solutions was measured with a surface tension balance (White Electric Instrument $\mathrm{Co}$ ).

\section{Electrospinning Process}

The blends were fed through a plastic syringe to the needle tip (20G diameter) and were electrospun under different voltages, produced by a high voltage source (Glassman High Voltage Inc.). The nanofibres were collected on a flat grounded collector covered with aluminum foil. The needle tip to collector distance was fixed at $12 \mathrm{~cm}$ at a horizontal orientation. The flow rate of the solutions was controlled by a syringe pump (Harvard Apparatus). The humidity in the electrospinning chamber was regulated by a constant dry air flow and monitored using a temperature and humidity meter ST-321. The temperature was monitored by the same device.

Finally, a Fluke 287 digital multimeter was interposed between the collector and the ground so as to allow the measurement of the current that is transferred by the jet on the collector for each experimental run.

The morphology of the electrospun mats was then examined with the use of Carl Zeiss (Leo) Scanning Electron Microscope (Model 1530VP). For the determination of the average diameter of the fibres the AxioVision software was used.

\section{Experimental Design}

A three-level full factorial design was used in order to determine the electrospinning window for the two specific PANI-PEO solutions in randomized runs. The maximum and minimum values for each parameter were determined from some initial scanning experiments that are not presented here. Especially for the voltage range, $13.5 \mathrm{kV}$ was used as higher value, as higher voltages resulted in short circuit of the device due to the high conductivity of the solution. As low voltage setting, the lowest one allowing Taylor cone formation was used, which would indicate the potential formation of a jet. 


\begin{tabular}{|c|c|c|c|}
\hline Run & Flow Rate (mL/hr) & $\%$ Relative Humidity & Voltage (kV) \\
\hline 1 & 3 & 32 & 13.5 \\
\hline 2 & 2 & 32 & 13.5 \\
\hline 3 & 1 & 32 & 13.5 \\
\hline 4 & 3 & 25 & 13.5 \\
\hline 5 & 2 & 25 & 13.5 \\
\hline 6 & 1 & 25 & 13.5 \\
\hline 7 & 3 & 18 & 13.5 \\
\hline 8 & 2 & 18 & 13.5 \\
\hline 9 & 1 & 18 & 13.5 \\
\hline 10 & 3 & 32 & 9.2 \\
\hline 11 & 2 & 32 & 9.2 \\
\hline 12 & 1 & 32 & 9.2 \\
\hline 13 & 3 & 25 & 9.2 \\
\hline 14 & 2 & 25 & 9.2 \\
\hline 15 & 1 & 25 & 9.2 \\
\hline 16 & 3 & 18 & 9.2 \\
\hline 17 & 2 & 18 & 9.2 \\
\hline 18 & 1 & 18 & 9.2 \\
\hline 19 & 3 & 32 & 5 \\
\hline 20 & 2 & 32 & 5 \\
\hline 21 & 1 & 32 & 5 \\
\hline 22 & 3 & 25 & 5 \\
\hline 23 & 2 & 25 & 5 \\
\hline 24 & 1 & 25 & 5 \\
\hline 25 & 3 & 18 & 5 \\
\hline 26 & 2 & 18 & 5 \\
\hline 27 & 1 & 18 & 5 \\
\hline
\end{tabular}

\section{Results and Discussion}

The effect of the parameters explored in this study (flow rate, applied voltage, ambient humidity, doping level) will be discussed with regards to the morphology of the nanofibrous structure, and the nanofibre diameter distribution. The electrospinning window will be defined and analyzed and finally the data obtained during this study will be fitted in an existing mathematical model to compare the behavior of the conductive polymer used in this study with the behavior of the non conductive polymers reported in the literature and determine the effect of polymer conductivity on the jet behavior and the diameter distribution. 


\section{Morphology}

The combined effects of the examined parameters on the fibre morphology show that the prevailing phenomena during electrospinning are related to the conductive character of the polymer.

\section{Effect of flow rate}

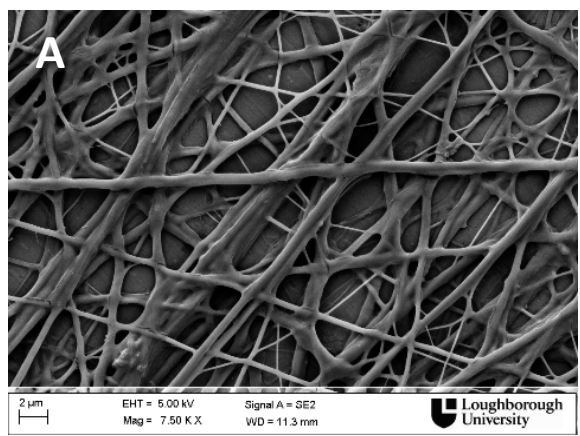

$3 \mathrm{~mL} / \mathrm{hr}, 13.5 \mathrm{kV}, 18 \% \mathrm{RH}$

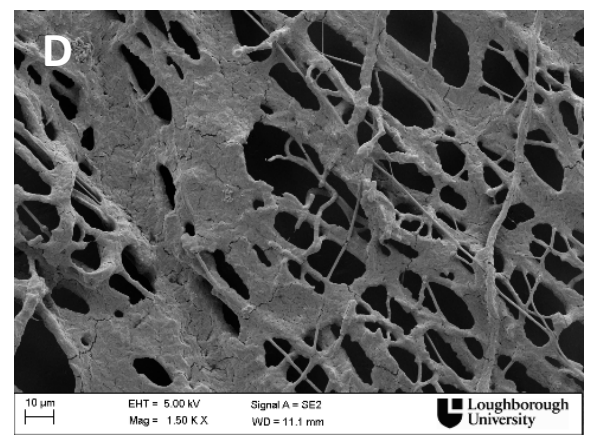

$3 \mathrm{~mL} / \mathrm{hr}, 9.2 \mathrm{kV}, 25 \% \mathrm{RH}$

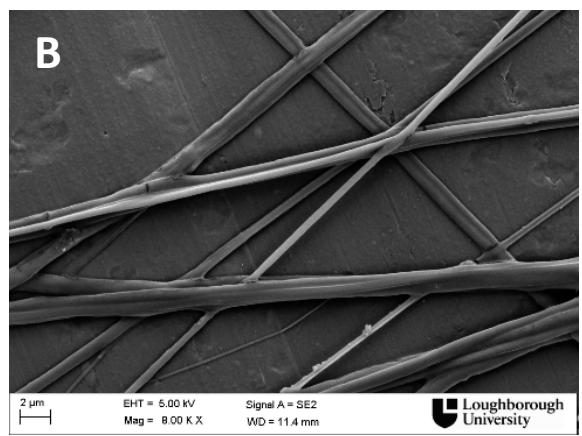

$2 \mathrm{~mL} / \mathrm{hr}, 13.5 \mathrm{kV}, 18 \% \mathrm{RH}$

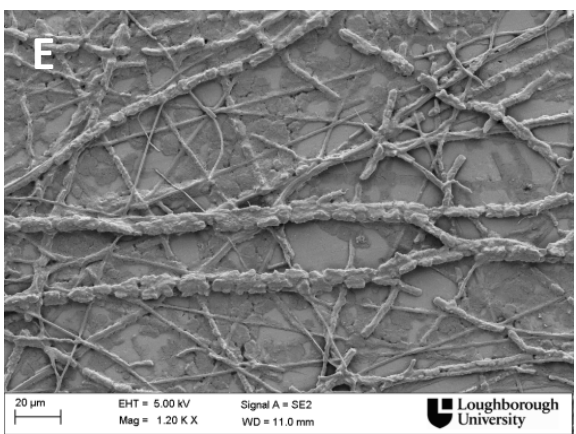

$2 \mathrm{~mL} / \mathrm{hr}, 9.2 \mathrm{kV}, 25 \% \mathrm{RH}$

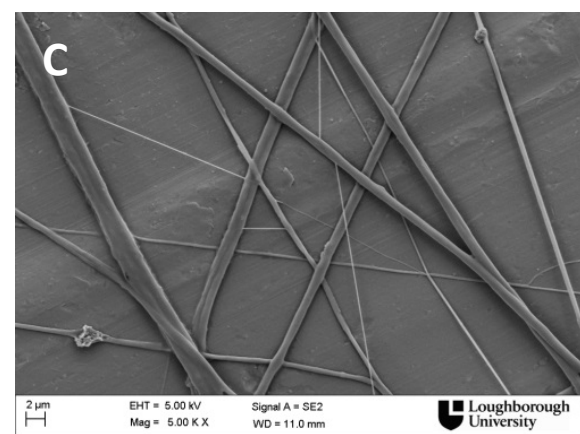

$1 \mathrm{~mL} / \mathrm{hr}, 13.5 \mathrm{kV}, 18 \% \mathrm{RH}$

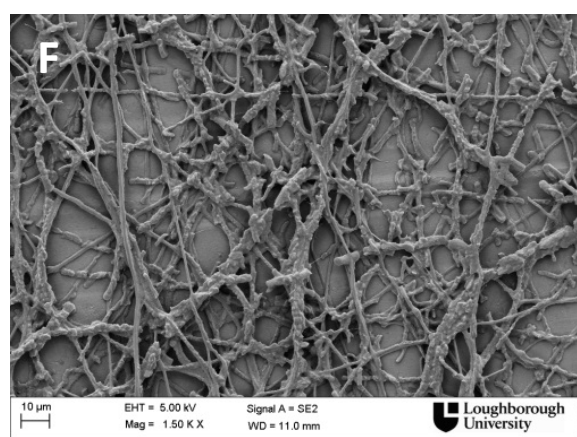

$1 \mathrm{~mL} / \mathrm{hr}, 9.2 \mathrm{kV}, 25 \% \mathrm{RH}$

Figure. 1: FEGSEM images for the comparison of flow rate variation on the nanofibre morphology

In terms of flow rate, the FEG SEM images obtained (Fig. 1) show a trend that was expected, based on the literature. Higher flow rates generally resulted in higher productivity and in some cases the nanofibres were not completely dry when they reached the collector. This was observed for different voltages and different levels of relative humidity, and could be attributed to the fact that, longer time would be required for the solvent to fully evaporate at higher flow rates and constant tip to collector distance. However, when the highest value of voltage was applied, the process became more stable at high flow rates. Low flow rates resulted in the formation of some very fine fibres, as shown in Fig 1.C, probably due to the high surface charge density which may have caused splitting of the jet [19]. 


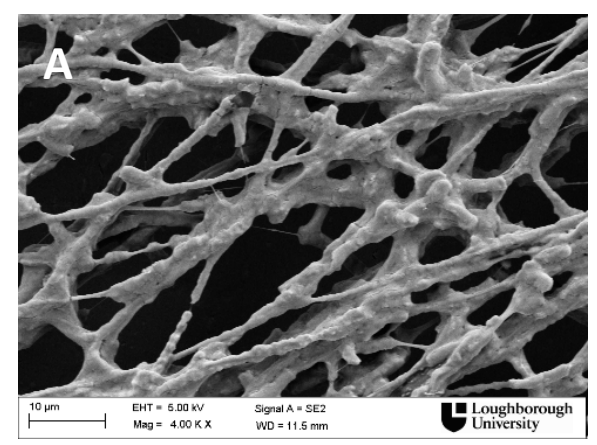

$1 \mathrm{~mL} / \mathrm{hr}, 5 \mathrm{kV}, 32 \% \mathrm{RH}$

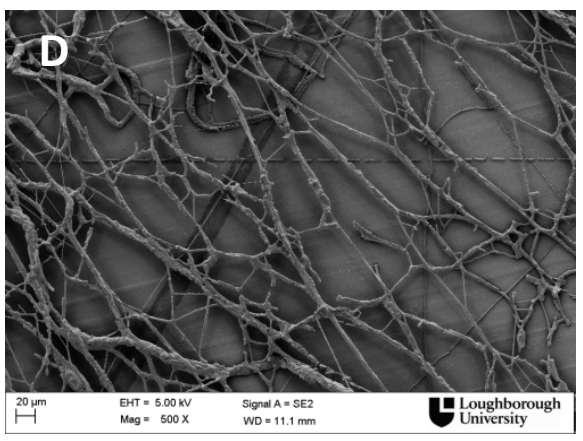

$3 \mathrm{~mL} / \mathrm{hr}, 13.5 \mathrm{kV}, 32 \% \mathrm{RH}$

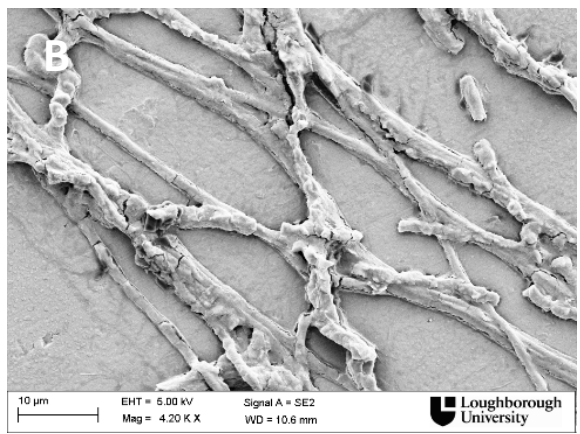

$1 \mathrm{~mL} / \mathrm{hr}, 5 \mathrm{kV}, 25 \% \mathrm{RH}$



$3 \mathrm{~mL} / \mathrm{hr}, 13.5 \mathrm{kV}, 25 \% \mathrm{RH}$

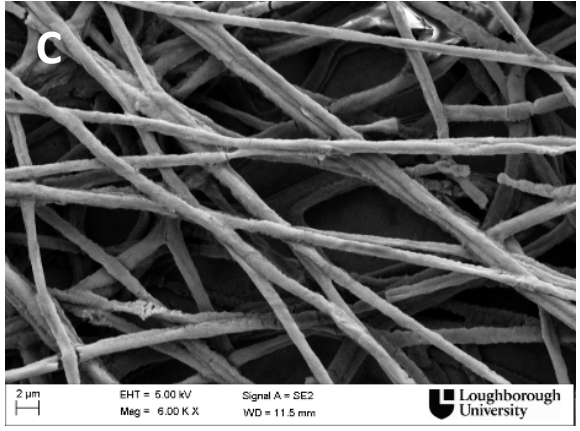

$1 \mathrm{~mL} / \mathrm{hr}, 5 \mathrm{kV}, 18 \% \mathrm{RH}$

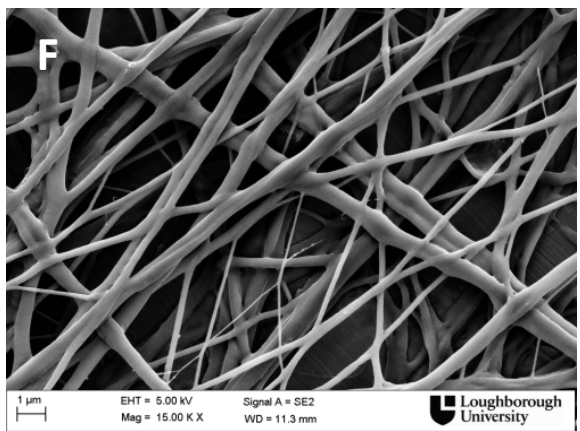

$3 \mathrm{~mL} / \mathrm{hr}, 13.5 \mathrm{kV}, 18 \% \mathrm{RH}$

Fig. 2: FEGSEM images showing the effect of relative humidity variation on the nanofibre morphology

Humidity was found to play a crucial role in electrospinnability and mat morphology. As it is shown on the SEM images in figure 2, only low ambient humidity allows the formation of defect free nanofibres throughout the whole range of applicable voltages. When the humidity increases to $25 \%$, the fibres start to break and irregular and uneven surfaces are formed, whereas electrospinning was not feasible at relative humidity higher than $40 \%$. The roughness of the surface occurring at higher humidity (fig 2.A), could be explained by phase separation that potentially occurred during the jet stretching caused by the absorption of water vapour by PEO. The electrospun solution consists of PEO which is a highly hydrophilic polymer, polyaniline which is insoluble in water and chloroform which is immiscible with water, therefore water absorption by the PEO could result in phase separation, precipitation of PANI and hence in uneven and rough nanofibre surface.

Our observations could be directly compared with Knopf's, who studied the effect of variation of environmental humidity during electrospinning of PEO solution in the same solvent. That study showed that relative humidity didn't have an effect on the electrospinning process or nanofibre morphology. On the contrary, in this case, the addition of polyaniline in the same PEO-chloroform solution resulted in humidity becoming a major factor for electrospinning [20]. The strong dependence of electrospinnability on the humidity for PANI solutions, except from the insolubility of PANI in water, could also be attributed to its conductivity. Higher humidity can remove surface charges from the jet or the solution surface at a higher rate, resulting in an increased discharge of the solution surface, which can either hinder the formation of a jet and impede electrospinnability or cause defects on the fibre surface. Angammana and Jayaram showed that the solution conductivity and the average jet current are closely related, with the jet current initially increasing for increasing solution conductivity and then decreasing with a further increase at solution conductivity. This was attributed to the decrease of the tangential electric field occurring at increased conductivity which 
causes the electrostatic force along the surface of the fluid to diminish [9]. Therefore, the high conductivity of PANI could lead to a decrease of electric field. This, combined with the increased discharge rate of the jet at higher humidity, gives rise to synergistic effects that exert very strong effects on PANI solutions rendering them very sensitive to humidity effects and set the relative humidity threshold for electrospinnability lower than for most polymers. This suggests that polymer conductivity can significantly affect the electrospinnability of a polymer solution in relationship to humidity variations. It can be suggested that by increasing the environmental humidity there is a charge unbalance taking place on the polymer jet, as charges can be exchanged from the polymer itself to the surrounding water vapors more easily than in the case of a non conductive polymer solution. The conductivity of the polymer might be facilitating this way, the transfer of charges from the center of the jet towards the surface, thus accelerating the discharge rate and rendering the impact of surrounding water vapors more important.

\section{Effect of voltage}

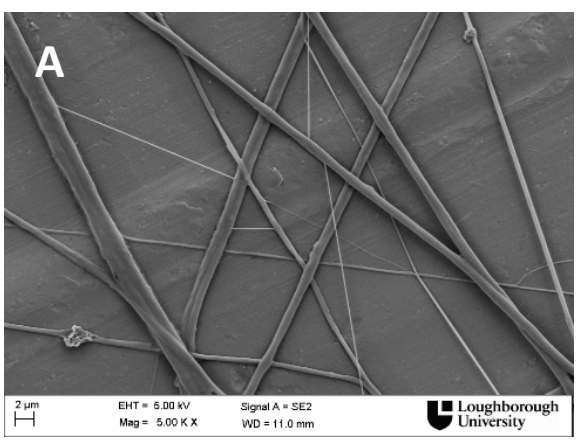

$1 \mathrm{~mL} / \mathrm{hr}, 18 \% \mathrm{RH}, 13.5 \mathrm{kV}$

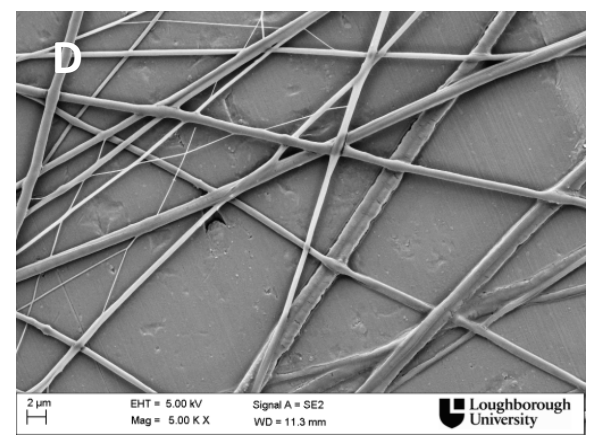

$2 \mathrm{~mL} / \mathrm{hr}, 18 \% \mathrm{RH}, 13.5 \mathrm{kV}$

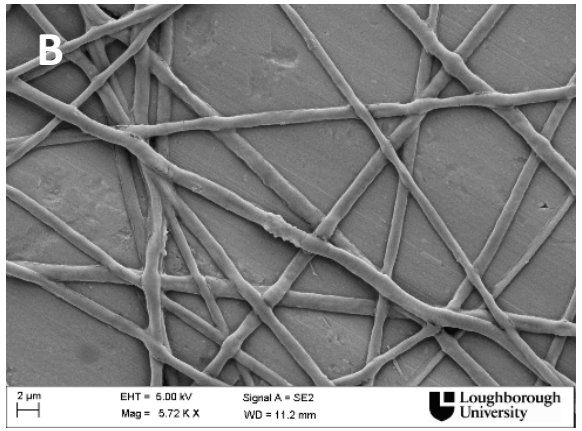

$1 \mathrm{~mL} / \mathrm{hr}, 18 \% \mathrm{RH}, 9.2 \mathrm{kV}$

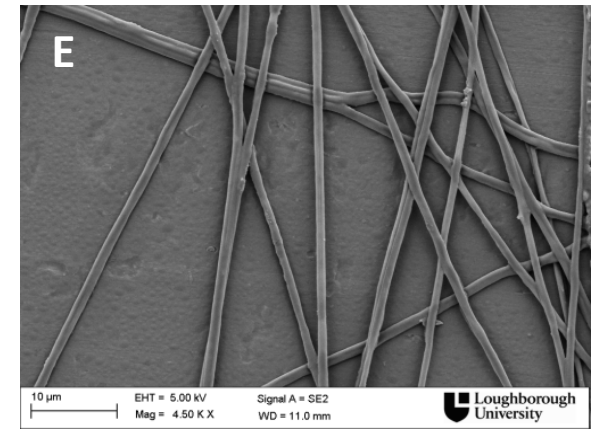

$2 \mathrm{~mL} . \mathrm{hr}, 18 \% \mathrm{RH}, 9.2 \mathrm{kV}$

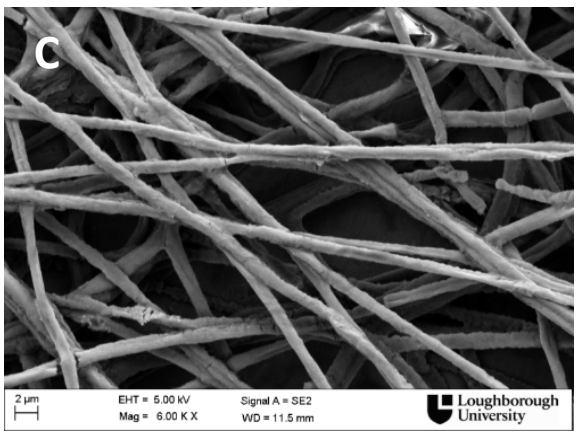

$1 \mathrm{~mL} / \mathrm{hr}, 18 \% \mathrm{RH}, 5 \mathrm{kV}$

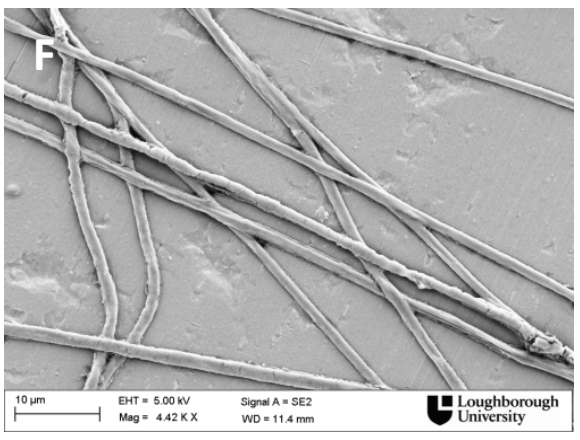

$2 \mathrm{~mL} / \mathrm{hr}, 18 \% \mathrm{RH}, 5 \mathrm{kV}$

Fig. 3: FEGSEM images for the comparison of applied voltage variation on the nanofibre morphology

As a general trend it seems that higher voltages produce thinner nanofibres but also a bigger diversity in diameters and a broader diameter distribution which will be discussed in the next section. Especially at $13,5 \mathrm{kV}$, for all flow rates, there was a significant percentage of very thin nanofibres within the mat, as shown on fig. 3 , indicating splitting of the jet. It has been reported that the jet may undergo splitting into multiple subjets in a process known as splaying or branching. This happens as a result of changes occurring in the shape, and charge per unit area of the jet during its elongation and the solvent's evaporation. The balance between the surface tension and the electrical forces may be 
shifted, usually, if the excess charge density on the surface of the jet is high causing undulations on the jet. These undulations may grow big enough to cause instability and then, in order to regain balance and to reduce the local charge per unit area, branches are initiated outward of the spinning jet. The formation of branches in jets and fibres usually occurs in more concentrated and viscous solutions and also when relatively high electric fields are applied [19,21].

It is also observed, that provided a low humidity, all the range of applied voltages results in defect free fibres indicating that the applied voltage is not a major factor affecting nanofibre formation.

\section{Effect of doping level}

The $20 \%$ doped solution was proved to be inappropriate for electrospinning as the inadequate doping rendered the solubility of the polyaniline in chloroform extremely low, and after the filtration there was practically no polyaniline left in the solution.

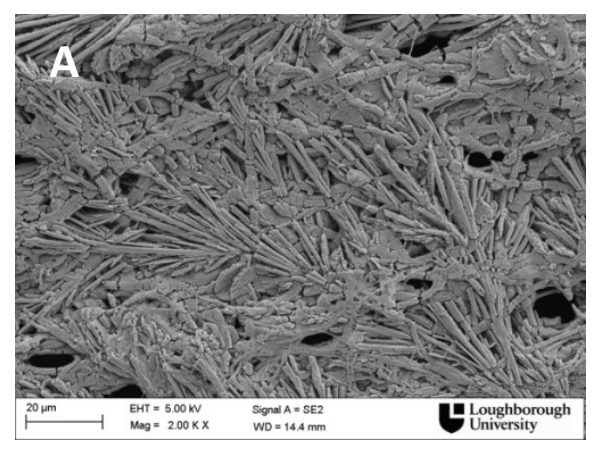

$1 \mathrm{~mL} / \mathrm{hr}, 9.2 \mathrm{kV}, 18 \% \mathrm{RH}$

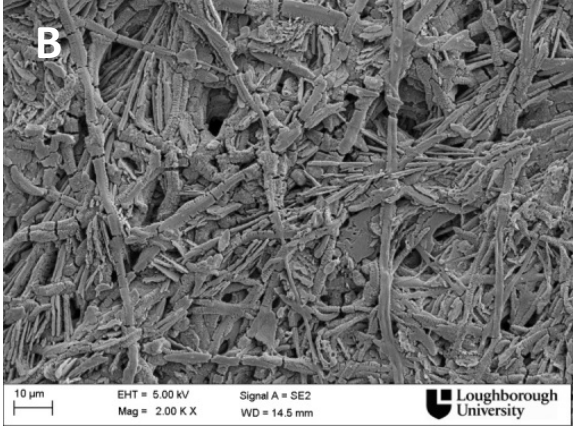

$3 \mathrm{~mL} / \mathrm{hr}, 9.2 \mathrm{kV}, 18 \% \mathrm{RH}$

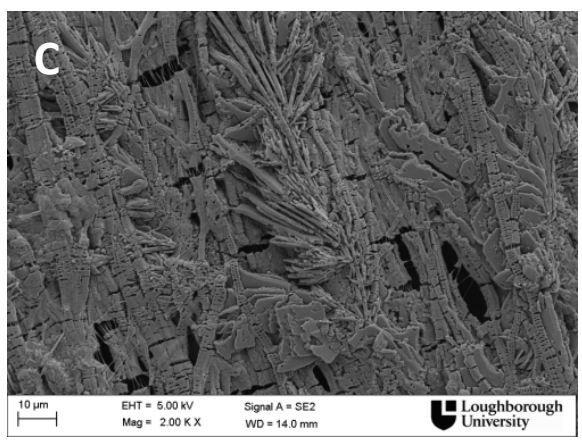

$3 \mathrm{~mL} / \mathrm{hr}, 13.5 \mathrm{kV}, 18 \% \mathrm{RH}$

Fig. 4: FEGSEM images of electrospun $100 \%$ CSA doped PANI- PEO solution under various process parameters at $18 \% \mathrm{RH}$

The solution containing PAni doped at a greater extent (100\%) produced structures of very high crystallinity and orientation but not nanofibrous mats. The experimental design that was used covers low, medium, and high values of flow rate, voltage and environmental humidity, so it can safely be concluded that the PANI (100\%doped) - PEO solution $1,8 \% \mathrm{w} / \mathrm{v}$ is not electrospinnable. Typical samples of the mats produced are shown on figure 4 and they reveal a crystalline structure of the produced mats. The amount of CSA that was added in order to increase the doping level seemed to cause the formation of crystalline structures during spinning that had a certain orientation as shown in fig 4. It has indeed been proven in the literature that casting of CSA doped polyaniline solutions results in crystalline structures as the acid hydrogen ion bonds to the imine sites of the polymer chain [22-24]. The reason why this morphology is obtained at high doping levels, is most likely due to the fact that the excess amount of CSA added in the solution increases the boiling point of the solvent, thus hindering the evaporation of the solvent within the range of parameters tested here, resulting in a wet mat, with no nanofibre structure but with the crystalline morphology reported at the above mentioned studies on film casting. 


\section{Electrospinning Window}

After analyzing the FEGSEM pictures obtained, the following charts were created illustrating the electrospinning windows of the $60 \%$ doped PANI- PEO solution.

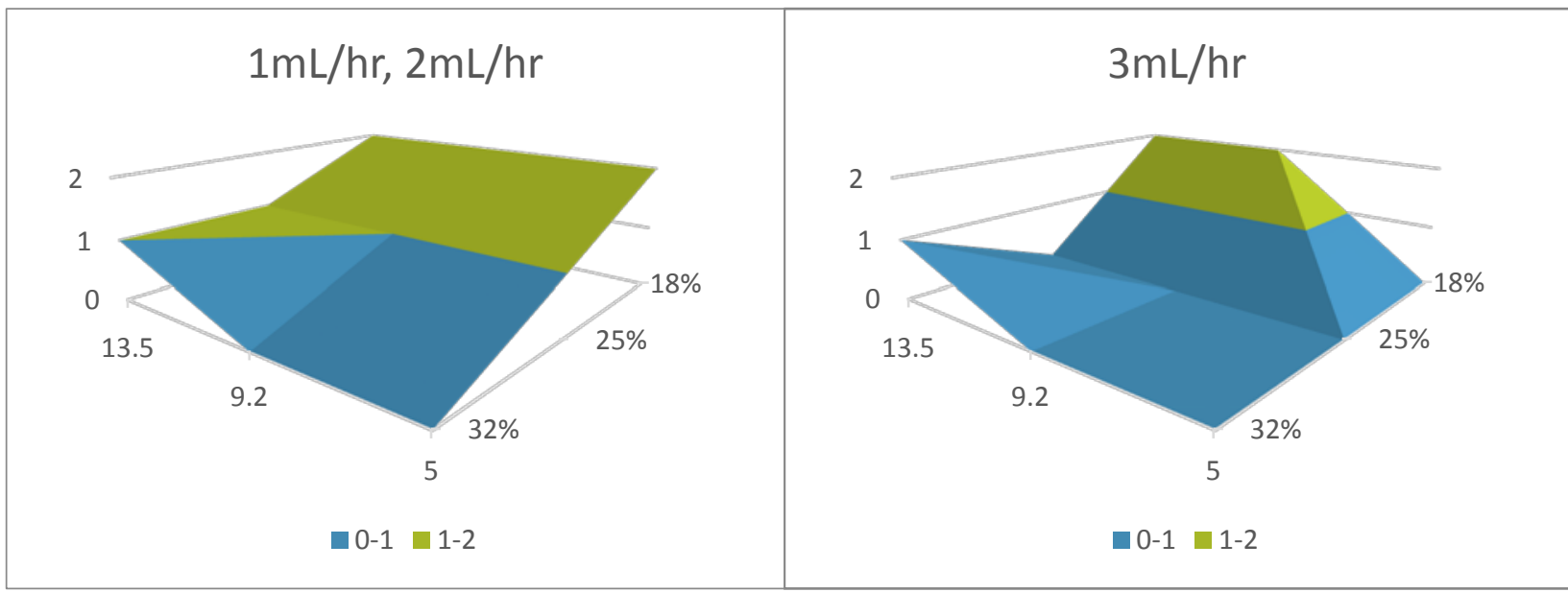

Fig. 5: Electrospinning window of $60 \%$ doped PANI-PEO solution, grouped by the flow rate, a) 1 and $2 \mathrm{~mL} / \mathrm{h}$, b) $3 \mathrm{~mL} / \mathrm{h}$. The coding is as follows: 0 : electrospinning was not possible/no fibres collected, 1 : electrospinning was possible - fibre-like morphology but with defects (beaded fibres, broken fibres, wet mat), 2: good nanofibre morphology

The electrospinning window at a flow rate of $1 \mathrm{~mL} / \mathrm{hr}$ is identical to the one at flow rate of $2 \mathrm{~mL} / \mathrm{hr}$, suggesting that although the flow rate affects the process, it is not the key parameter in terms of electrospinnability. Green areas, in figure 5, depict the conditions where a stable jet formed and defect free fibres were produced (level 1-2) and the blue areas depict the conditions where electrospinning was hindered, thus producing fibres with defects or where electrospinning could not be conducted at all (level $0-1$ ). It is shown that at flow rates of 1 and $2 \mathrm{~mL} / \mathrm{hr}$, the electrospinning window is wider than at $3 \mathrm{~mL} / \mathrm{hr}$, allowing formation of nanofibres at a wider range of conditions. Similarly when $18 \% \mathrm{RH}$ is applied the electrospinning window is bigger allowing the formation of nanofibres over a broader range of conditions and of combinations of the other two parameters (flow rate and voltage).

It is quite clear, that the role of humidity is crucial. It is almost only at low humidity that defect free nanofibres could be collected. This indicates that humidity higher than $20 \%$ may hinder the electrospinning process in a number of ways. At higher humidity, water vapor removes surface charges, that drive the jet elongation, leading to discharge of the jet and hence to smaller electrostatic forces between surface charges. This might hinder the formation of a Taylor cone or the extrusion of the jet from the Taylor cone, or lead to the formation of beads and defects on the fibres. Also, at higher humidity, water vapor can be absorbed by the PEO in the jet which can cause phase separation as PANI is not soluble in water. Finally, high humidity may result in slower solidification of the jet. 
For the combinations of these three parameters that resulted in the production of defect free nanofibrous mats, a further investigation regarding the nanofibres diameter was conducted to determine the effect of the process parameters on the mean diameter and diameter distribution. Results were based on measuring the diameter on 150 nanofibres for each sample and are summed up on the diagrams of figure 6.

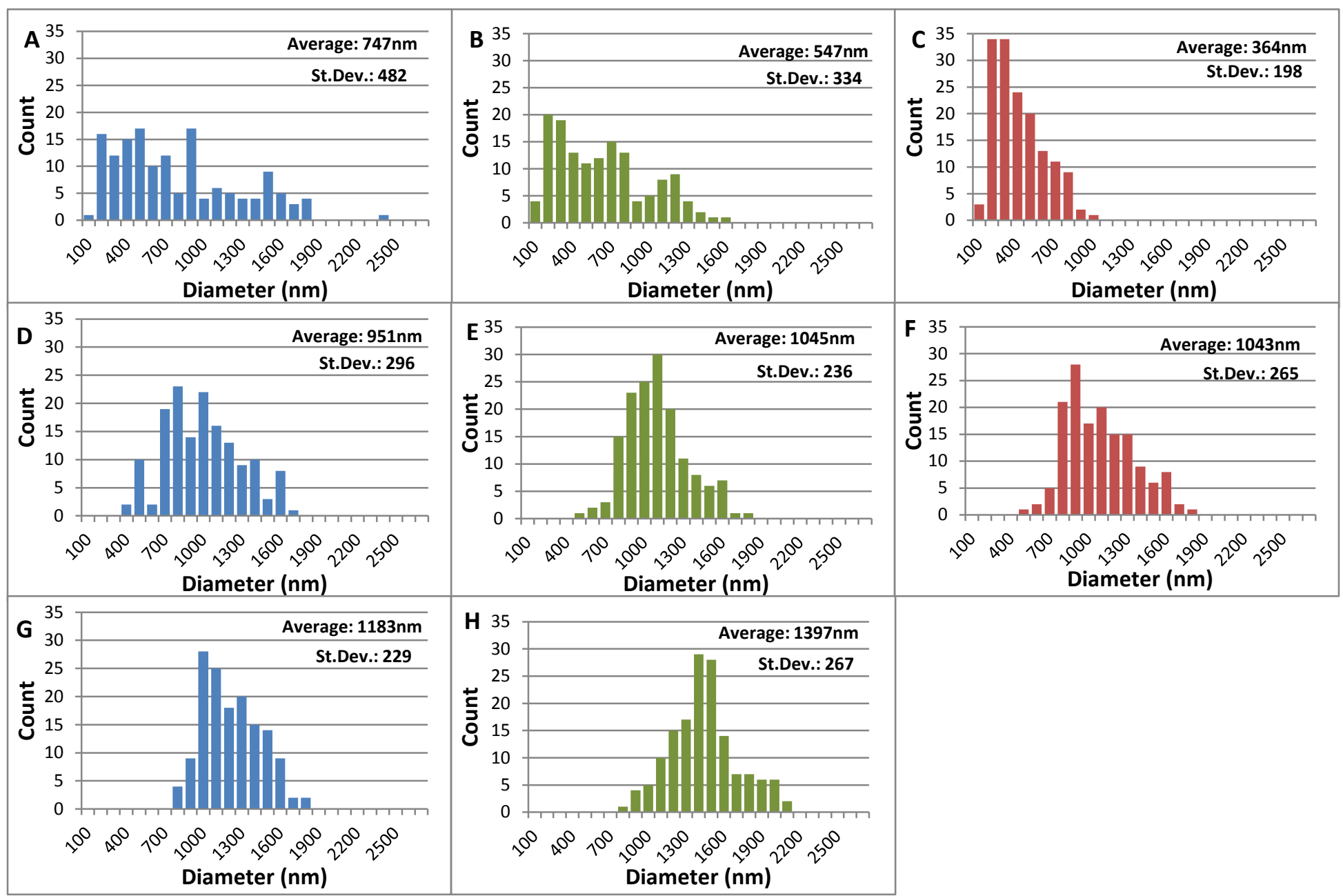

Figure 6: Diameter Distribution for electrospun mats at $18 \% \mathrm{RH}:$ A) $1 \mathrm{~mL} / \mathrm{hr}, 13.5 \mathrm{kV}$ B) $2 \mathrm{~mL} / \mathrm{hr}$, $13.5 \mathrm{kV}$ C) $3 \mathrm{~mL} / \mathrm{hr}, 13.5 \mathrm{kV}$, D) $1 \mathrm{~mL} / \mathrm{hr}, 9.2 \mathrm{kV}$ E) $2 \mathrm{~mL} / \mathrm{hr}, 9.2 \mathrm{kV}$ and F) ) $3 \mathrm{~mL} / \mathrm{hr}, 9.2 \mathrm{kV} \mathrm{G}) 1 \mathrm{~mL} / \mathrm{hr}, 5 \mathrm{kV}$ H) $2 \mathrm{~mL} / \mathrm{hr}, 5 \mathrm{kV}$

High voltage combined with low flow rate results in relatively thin nanofibres based on average diameter measurements, but the distribution range is very wide, indicating splitting of the jet, probably because of very high surface charge density. At constant voltage $(13,5 \mathrm{kV})$, as the flow rate increases (Fig 6. A-C), the mean diameter and the distribution range (or the width of the diameter distribution) decrease, which is shown by the graphs and the decreasing standard deviation values. At constant flow rate the diameter distribution shifts to larger diameters and becomes more narrow and uniform for decreasing voltage (Fig. 6.A, D, G) indicating that splitting of the fibres ceased. At lower voltage of $9.2 \mathrm{kV}$ (Fig 6.D-F) the mean diameters increase and the distributions shift to bigger diameters at all flow rates. The shape of the distribution also changes and becomes more uniform at flow rates of $1 \mathrm{~mL} / \mathrm{hr}$ and $2 \mathrm{ml} / \mathrm{hr}$ indicating a more stable process. The narrowest distribution was 
obtained at the applied conditions: [13.5kV, $3 \mathrm{~mL} / \mathrm{hr}, 18 \% \mathrm{RH}]$ followed by the $[2 \mathrm{~mL} / \mathrm{hr}, 9.2 \mathrm{kV} .18 \% \mathrm{RH}]$ and $[3 \mathrm{~mL} / \mathrm{hr}, 9.2 \mathrm{kV}, 18 \% \mathrm{RH}]$.

The combined effects of the process parameters on the mean fibre diameters are shown in figures 7 and 8 , where the variation of the average diameter in relation to the viscosity and the flow rate is shown respectively.

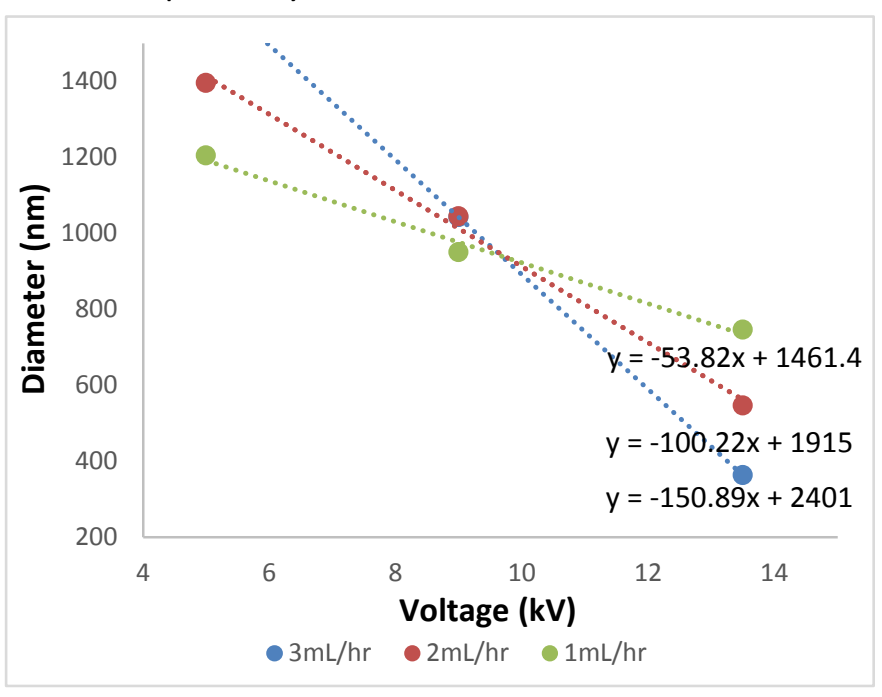

Fig 7: Effect of the applied voltage on the nanofibre diameter

Regarding the effect of the voltage, figure 7 , shows consistency on the way the voltage affects nanofibre diameter. Application of higher voltage, in general, produces thinner nanofibres at all flow rates. The impact of this though is more pronounced at higher flow rates, as the slopes of the linear trend lines indicate, almost doubling for each different flow rate used. The reason that the impact of voltage is more pronounced at higher flow rates could be understood considering the dual effect of the voltage on the jet. Firstly, higher voltage provides more thermal energy to the jet and hence accelerates the solvent evaporation leading to faster solidification of the jet. Secondly, higher voltage provides higher surface charge density and stronger repulsion between the surface charges, hence more stretching of the jet. The combination of these two factors, result in a different slope in figure 7 for different conditions. At low flow rates, faster jet solidification prevented further stretching of the jet compared to higher flow rates, where the jet solidified slower and stretching occurred to a greater extent, hence the second effect was more pronounced. These findings contribute to clarifying the controversy encountered in the literature about the effect of the voltage on various solutions and under various electrospinning conditions.

With regard to the flow rate, its effect on the average diameters depends on the voltage. At a higher voltage, the average diameter decreases as the flow rate increases. At medium voltage, it doesn't change significantly, meaning that the effect of the flow rate is counterbalanced by the voltage increase resulting in a steady diameter, and at low voltage the relationship seems to get reversed. Although at high flow rate it wasn't possible to electrospin at $5 \mathrm{kV}$ and therefore the average diameter could not be calculated, the trend is evident. This opposes the majority of findings reported in the literature regarding the effect of the flow rate on the final nanofibre diameter $[10,25]$. 


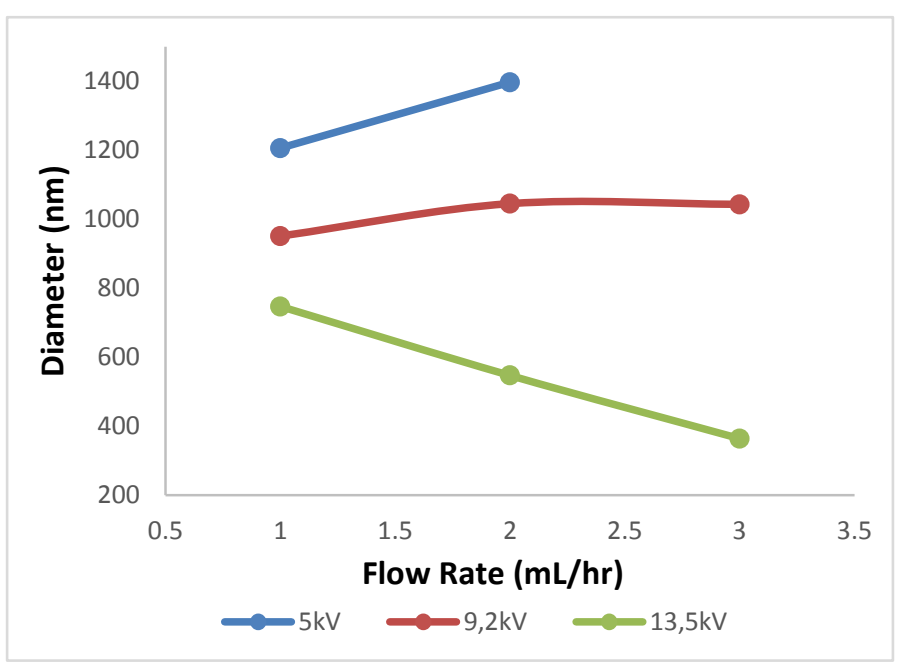

Fig 8: Effect of flow rate on the nanofibre diameter

These observations could be explained by taking into consideration the effect of the voltage on the evaporation rate of the solvent and the jet's solidification rate. Application of higher voltage causes the temperature of the jet to increase, and therefore the solvent to evaporate faster. Fast solvent evaporation means fast jet solidification which in turn prevents further stretching of the jet leading to bigger fibre diameters at low flow rates. As the flow rate increases at the same high voltage, the jet solidifies slower and hence it is subjected to more stretching leading to the formation of finer fibres. This explains why, at high voltage, the diameter decreases with increasing flow rate. Please note that these findings also depend on the volatility of the solvent. At low voltage however, different phenomena prevail. At low voltage the strength of the electric field is not sufficient to cause further stretching of the jet for increased flow rate, hence as the flow rate increases the fibre diameter also increases. At medium voltage values, the combined action of the two competing phenomena lead to insignificant differences in the jet diameter with increasing flow rate.

\section{Data fitting}

For the combination of the experiment parameters that resulted in measurable nanofibres, we examined how the data fit on the diameter prediction model proposed by Fridrikh et al.[7]

$h=\left(\gamma \bar{\varepsilon} \frac{Q^{2}}{I^{2}} \frac{2}{\pi(2 \ln \chi-3)}\right)^{1 / 3}$

This model predicts the terminal diameter of the jet (h) as a function of $\varepsilon$ (solvent dielectric permittivity), Q (flow rate) and I (current measured on collector); where $\chi$ is the dimensionless wavelength of the instability responsible for the normal displacements and here it is considered to equal 100. The exact value is not critical, since Inx varies slowly [7][26].

The terminal diameter of the jet is defined as the diameter of the jet at the late stages of whipping, where the dramatic stretching of the jet due to the whipping instability ceases. equation (1) neglects elastic effects and fluid evaporation, and also assumes minimal jet thinning after the saturation of the whipping instability. Fridrikh et al. tested these assumptions by measuring the 
diameters of electrospun fibres obtained over a wide range of external conditions and from different polymer solutions (PCL in methanol, PEO in water and PANI in N, N-dimethyl formamide). [7]

Equation 1 was used to calculate all theoretical diameters for the electrospun $60 \%$ doped PANI-PEO solutions. The surface tension of the solution was measured to be $30.2 \mathrm{mN} / \mathrm{m}$, the dielectric constant of chloroform is found in the literature [27] and the current values were measured for each of the process parameters combination at $18 \% \mathrm{RH}$. Figure 9 presents how the predicted and measured nanofibre diameters compare. The application of the proposed model resulted in an underprediction of the resulting nanofibres for all combinations of parameters except for the data points corresponding to high flow rate, which show good agreement with the model.

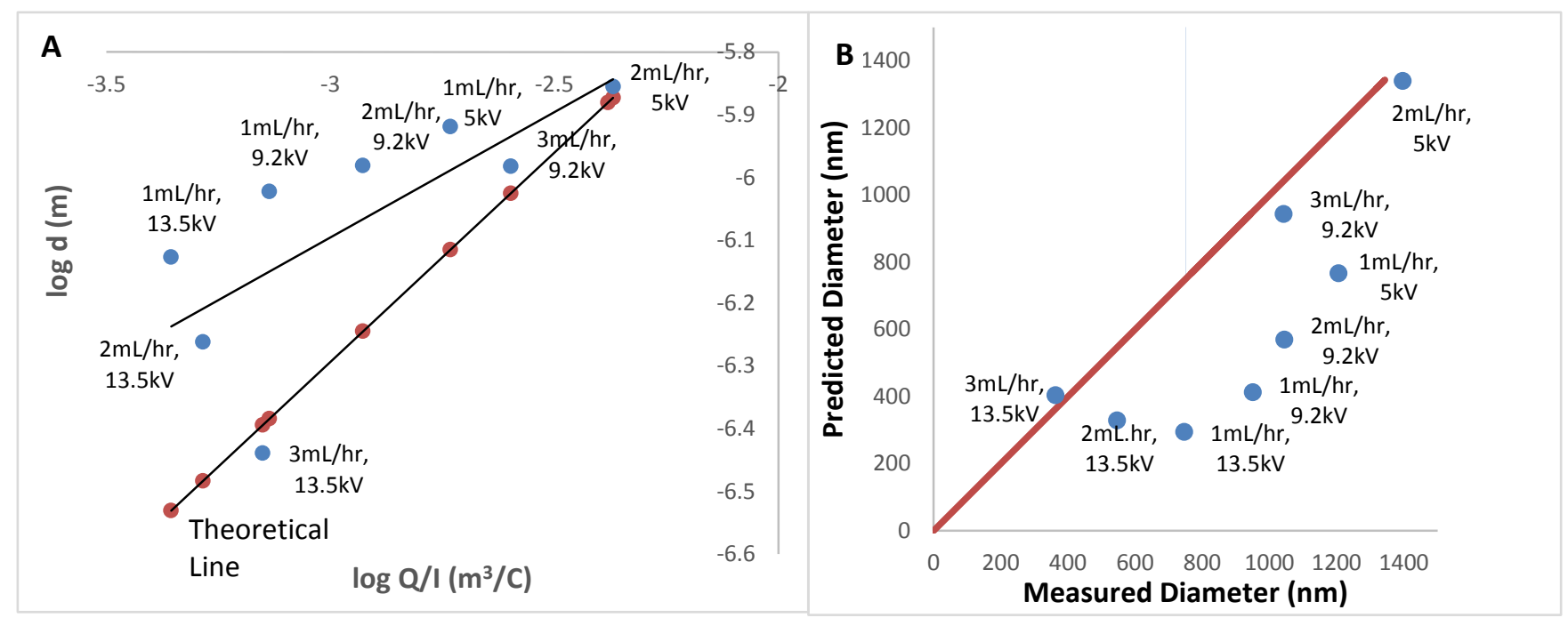

The deviation of the predicted from the actual diameters could be attributed to the conductivity of the polymer, the complex relationship between conductivity, jet current and fibre diameter, and the evaporation rate of the solvent. The solutions studied and reported in the literature refer to nonconductive polymers, where the conductivity of the jet is mainly due to the conductivity of the solvent. The conductivity affects the distribution of surface charges and the tangential electric field forces exerted on the jet. As the solvent evaporates the distribution of surface charges on the jet surface will be different for a conductive polymer compared to a non-conductive one resulting in a deviation from the expected values. The jet current increases with conductivity within certain range and then it decreases with conductivity [9]. This complex relationship between conductivity, jet current and the fibre diameter has not been taken into account. According to the model the relationship between jet current and fibre diameter is monotonic. However Angammana et al showed that the fibre diameter decreases with increasing jet current within a range of conductivities but increases with increasing jet current for higher conductivities. Figure 9A shows that for the same $\mathrm{Q} / \mathrm{I}$ the experimental data agree with the predicted behavior at high flow rates but deviate at low flow rates. This deviation may be attributed to a great extent to the effect of solvent evaporation which can be more pronounced at lower flow rates. 


\section{Conclusions}

Humidity was shown to be the -most important parameter affecting electrospinning and defining the electrospinning window for PANI solutions. Only at very low humidity, electrospinning was feasible, indicating that for conductive polymers, the effect of humidity may be significantly greater compared to that of non conductive polymers. To our knowledge this is the first study examining the importance of environmental humidity on electrospinning of a conductive polymer in an organic solvent. Higher ambient humidity disrupted the electrospinning process and resulted in irregular and rough fibre surfaces.

Flow rate and strength of electric field were found to have an impact on the final nanofibre diameter. Higher values of applied voltage resulted in thinner nanofibres at all flow rates. But when high voltage was combined with low flow rate, the phenomenon of branching of the jet was observed, resulting in broader diameter distribution. The impact of the flow rate was found to be dependent on the applied voltage. At high applied voltage, the nanofibre diameter decreases with the increase of flow rate while at low voltage the opposite trend is observed. At medium values of applied voltage the effect of the flow rate is counter balanced by the increased voltage. Low doping level rendered the polyaniline insoluble in chloroform and a high one caused the doping acid to crystallize and totally alter the morphology of the final mat. Finally, the diameter data were fitted on Fridrikh's et al. prediction model showed under prediction of the diameters in most cases probably due to the effects of the solvent evaporation and conductivity of the polymer blend.

Acknowledgements: The authors would like to thank the Doctoral Training Centre and the Graduate School of Loughborough University for funding this work.

\section{References}

[1] Svirskis D, Travas-Sejdic J, Rodgers A, Garg S. Electrochemically controlled drug delivery based on intrinsically conductive polymers. J Control Release 2010;146:6-15.

doi:10.1016/j.jconrel.2010.03.023.

[2] Guimard NK, Gomez N, Schmidt CE. Conductive polymers in biomedical engineering. Prog Polym Sci 2007;32:876-921. doi:10.1016/j.progpolymsci.2007.05.012.

[3] Das TK, Prusty S. Review on Conductive Polymers and Their Applications. Polym Plast Technol Eng 2012;51:1487-500. doi:10.1080/03602559.2012.710697.

[4] Li C, Chartuprayoon N, Bosze W, Low K, Lee KH, Nam J, et al. Electrospun Polyaniline/Poly(ethylene oxide) Composite Nanofibres Based Gas Sensor. Electroanalysis 2014;26:711-22. doi:10.1002/elan.201300641.

[5] Cao Y, Smith P, Alan JH. Counter-ion induced processibility of conductive polyaniline and of conductive polyblends of polyaniline in bulk polymers 1992;48:91-7.

[6] Moayeri A, Ajji A. Fabrication of polyaniline / poly ( ethylene oxide )/ non-covalently functionalized graphene nanofibres via electrospinning 2015;200:7-15. 
[7] Fridrikh S, Yu J, Brenner M, Rutledge G. Controlling the Fibre Diameter during Electrospinning. Phys Rev Lett 2003;90:144502. doi:10.1103/PhysRevLett.90.144502.

[8] Hohman MM, Shin M, Rutledge G, Brenner MP. Electrospinning and electrically forced jets. II. Applications. Phys Fluids 2001;13:2221. doi:10.1063/1.1384013.

[9] Angammana CJ, Member S, Jayaram SH. Analysis of the Effects of Solution Conductivity on Electrospinning Process and Fibre Morphology 2011;47:1109-17.

[10] Thompson CJ, Chase GG, Yarin a. L, Reneker DH. Effects of parameters on nanofibre diameter determined from electrospinning model. Polymer (Guildf) 2007;48:6913-22.

doi:10.1016/j.polymer.2007.09.017.

[11] Rafiei S, Maghsoodloo S, Noroozi B, Mottaghitalab V, Haghi AK. CELLULOSE CHEMISTRY AND TECHNOLOGY MATHEMATICAL MODELING IN ELECTROSPINNING PROCESS OF NANOFIBRES : A DETAILED REVIEW 2013;47:323-38.

[12] Vrieze S, Camp T, Nelvig a., Hagström B, Westbroek P, Clerck K. The effect of temperature and humidity on electrospinning. J Mater Sci 2008;44:1357-62. doi:10.1007/s10853-0083010-6.

[13] Ahmed FE, Lalia BS, Hashaikeh R. A review on electrospinning for membrane fabrication: Challenges and applications. Desalination 2015;356:15-30. doi:10.1016/j.desal.2014.09.033.

[14] Pelipenko J, Kristl J, Janković B, Baumgartner S, Kocbek P. The impact of relative humidity during electrospinning on the morphology and mechanical properties of nanofibres. Int J Pharm 2013;456:125-34. doi:10.1016/j.ijpharm.2013.07.078.

[15] Nezarati RM, Eifert MB, Cosgriff-hernandez E. Effects of Humidity and Solution Viscosity on Electrospun Fibre Morphology 2013;19:810-9. doi:10.1089/ten.tec.2012.0671.

[16] Tripatanasuwan S, Zhong Z, Reneker DH. Effect of evaporation and solidification of the charged jet in electrospinning of poly(ethylene oxide) aqueous solution. Polymer (Guildf) 2007;48:5742-6. doi:10.1016/j.polymer.2007.07.045.

[17] Li S, Zheng G, Wang X, Chen Y, Wu D, Sun D. Improved Electrical Conductivity of PANI / PEO Polymer via Electrospinning and its Application as NH 3 Gas Sensor n.d.;1:3-6.

[18] Li D, Xia Y. Electrospinning of Nanofibres: Reinventing the Wheel? Adv Mater 2004;16:115170. doi:10.1002/adma.200400719.

[19] Garg K, Bowlin GL. Electrospinning jets and nanofibrous structures. Biomicrofluidics 2011;5:13403. doi:10.1063/1.3567097.

[20] Knopf JA. Investigation of linear electrospinning jets. University of Delaware, 2009.

[21] Reneker DH, Yarin AL. Electrospinning jets and polymer nanofibres. Polymer (Guildf) 2008;49:2387-425. doi:10.1016/j.polymer.2008.02.002. 
[22] Saravanan S, Joseph Mathai C, Anantharaman MR, Venkatachalam S, Prabhakaran PV. Investigations on the electrical and structural properties of polyaniline doped with camphor sulphonic acid. J Phys Chem Solids 2006;67:1496-501. doi:10.1016/j.jpcs.2006.01.100.

[23] Magnuson M, Guo J, Butorin S, Agui A, Såthe C, Nordgren J, et al. The electronic structure of polyaniline and doped phases studied by soft X-ray absorption and emission spectroscopies 1999:4756-61.

[24] Ćirić-Marjanović G. Recent advances in polyaniline research: Polymerization mechanisms, structural aspects, properties and applications. Synth Met 2013;177:1-47. doi:10.1016/j.synthmet.2013.06.004.

[25] Pillay V, Dott C, Choonara YE, Tyagi C, Tomar L, Kumar P, et al. A Review of the Effect of Processing Variables on the Fabrication of Electrospun Nanofibres for Drug Delivery Applications. J Nanomater 2013;2013:1-22. doi:10.1155/2013/789289.

[26] Hohman MM, Shin M, Rutledge G, Brenner MP. Electrospinning and electrically forced jets. I. Stability theory. Phys Fluids 2001;13:2201. doi:10.1063/1.1383791.

[27] A. Maryott ERS. Table of Dielectric Constants of Pure Liquids. National Bureau of Standards Circular 514; n.d. 